Ann. Génét. Sél. Anim., I978, 10 (I), I7-27.

\title{
Identification par électrophorèse d'hybrides de Salmonidés
}

\author{
R. GUYOMARD \\ Laboratoire de Physiologie des Poissons, I.N.R.A., \\ Centre national de Recherches zootechniques, \\ 78350 Jouy-en-Josas, France.
}

\section{Résumé}

Sept systèmes protéiques ont été étudiés chez cinq espèces de Salmonidés: 1'Omble de fontaine (Salvelinus fontinalis Mitchell), la Truite arc-en-ciel (Salmo gairdneri R.), la Truite fario (Salmo trutta L.), le Saumon coho (Onchorynchus kisutch Walbaum.) et le Saumon atlantique (Salmo salar L.)

La phosphoglucose isomerase (PGI) nous a permis de vérifier le caractère hybride des individus issus des croisements mâle Omble de fontaine $\times$ femelle Truite arc-en-ciel, mâle Truite fario $\times$ femelle Saumon atlantique, mâle Truite fario $\times$ femelle Truite arc-en-ciel, mâle Omble de fontaine $\times$ femelle Truite fario; les alevins issus de croisements mâle Saumon coho $\times$ femelle Truite arc-en-ciel sont également des hybrides, identifiables par les protéines anodiques du muscle.

\section{Introduction}

Chez les Salmonidés, le problème de l'identification des hybrides interspécifiques naturels ou issus d'insémination artificielle a été abordé par plusieurs auteurs (cf. revues bibliographiques de Schwartz, I972 et DANGEL, I973). Cependant, l'utilisation de caractères systématiques classiques comme le nombre de rayons osseux ou de branchiospines s'avère souvent inopérante, les distributions des caractères, chez l'hybride, chevauchant partiellement, dans la plupart des cas, les distributions parentales (SUzUKI, I973). Les fonctions discriminantes n'apportent que peu d'améliorations et le recours à des marqueurs mendeliens visibles, comme l'albinisme chez la Truite arc-en-ciel (Salmo gairdneri $R$.) est pratiquement limité à cette espèce.

L'analyse du polymorphisme protéique par électrophorèse s'est, par contre, révélée un outil puissant puisqu'il est souvent possible de trouver un système de protéines fonctionnelles ou structurales qui permette de différencier deux espèces et conduise chez l'hybride à un électrophorégramme représentant la sommation 
plus ou moins complète des différents composants protéiques parentaux (NymAN, r970).

Nous avons appliqué cette technique à l'étude de cinq croisements interspécifiques pour vérifier si les alevins produits étaient effectivement des hybrides ou s'ils pouvaient résulter d'un développement gynogénétique.

\section{Matériel et méthodes}

Les parents âgés de 2 à 4 ans proviennent de différentes piscicultures françaises. Les ovules de chaque femelle ont été fécondés par un mélange de volumes égaux de sperme de plusieurs mâles, en utilisant un dilueur d'insémination (solution minérale tamponnée; BILLARD et al., I973). Le nombre de géniteurs utilisés pour

\section{TABLEAU I}

Nombre d'individus examinés par espèce parentale et pour chaque croisement étudié Number of individus examined for every parental species and cross

\begin{tabular}{|c|c|c|c|c|}
\hline 8 & \multicolumn{2}{|c|}{ Truite arc-en-ciel } & Saumon Atlantique & Truite fario \\
\hline$\hat{0}$ & $\begin{array}{c}\text { Coho } \times \text { Arc } \\
\mathrm{N}_{1}=23 \\
\mathrm{~N}_{2}=\mathrm{I} 2\end{array}$ & $\begin{array}{c}\text { Autres } \\
\text { croisements } \\
\mathrm{N}_{1}=23 \\
\mathrm{~N}_{2}=10\end{array}$ & $\begin{array}{l}N_{1}=2 \\
N_{2}=18\end{array}$ & $\begin{array}{l}\mathrm{N}_{1}=\mathrm{I} 3 \\
\mathrm{~N}_{2}=\mathrm{I} 2\end{array}$ \\
\hline $\begin{array}{l}\text { Saumon coho: } \\
\qquad \mathrm{N}_{1}=\mathrm{I} 2 . . \\
\mathrm{N}_{2}=\mathrm{II} . .\end{array}$ & $\left\{\begin{array}{r}499 \\
399 \\
n=\end{array}\right\}$ & 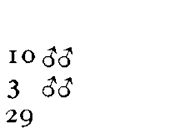 & & \\
\hline $\begin{array}{l}\text { Truite fario : } \\
\qquad \mathrm{N}_{1}=\mathrm{I} 4 . \\
\mathrm{N}_{2}=\mathrm{I} 2\end{array}$ & 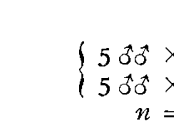 & $\begin{array}{l}12 \% 9 \\
4 \text { क } 99 \\
15\end{array}$ & $\begin{array}{c}399 \times 10 \\
n=13\end{array}$ & \\
\hline $\begin{array}{l}\text { Omble de fontaine } \\
\mathrm{N}_{1}=2 \mathrm{I} . \\
\mathrm{N}_{2}=\mathrm{I}\end{array}$ & $\begin{array}{r}4 \text { 웅 } \\
n=\end{array}$ & 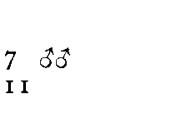 & & $\begin{array}{c}299 \times 730 \\
n=19\end{array}$ \\
\hline
\end{tabular}

- Pour chaque espèce parentale (mâle ou femelle) :

$\mathrm{N}_{1}=$ nombre d'adultes examinés

$\mathrm{N}_{\mathrm{2}}=$ nombre d'alevins examinés

- Pour chaque croisement est indiqué :

- le nombre d'hybrides étudiés $(n)$,

- le nombre de mâles et femelles utilisés pour produire les hybrides ( 1 ou 2 séries ont été réalisées selon les cas). 


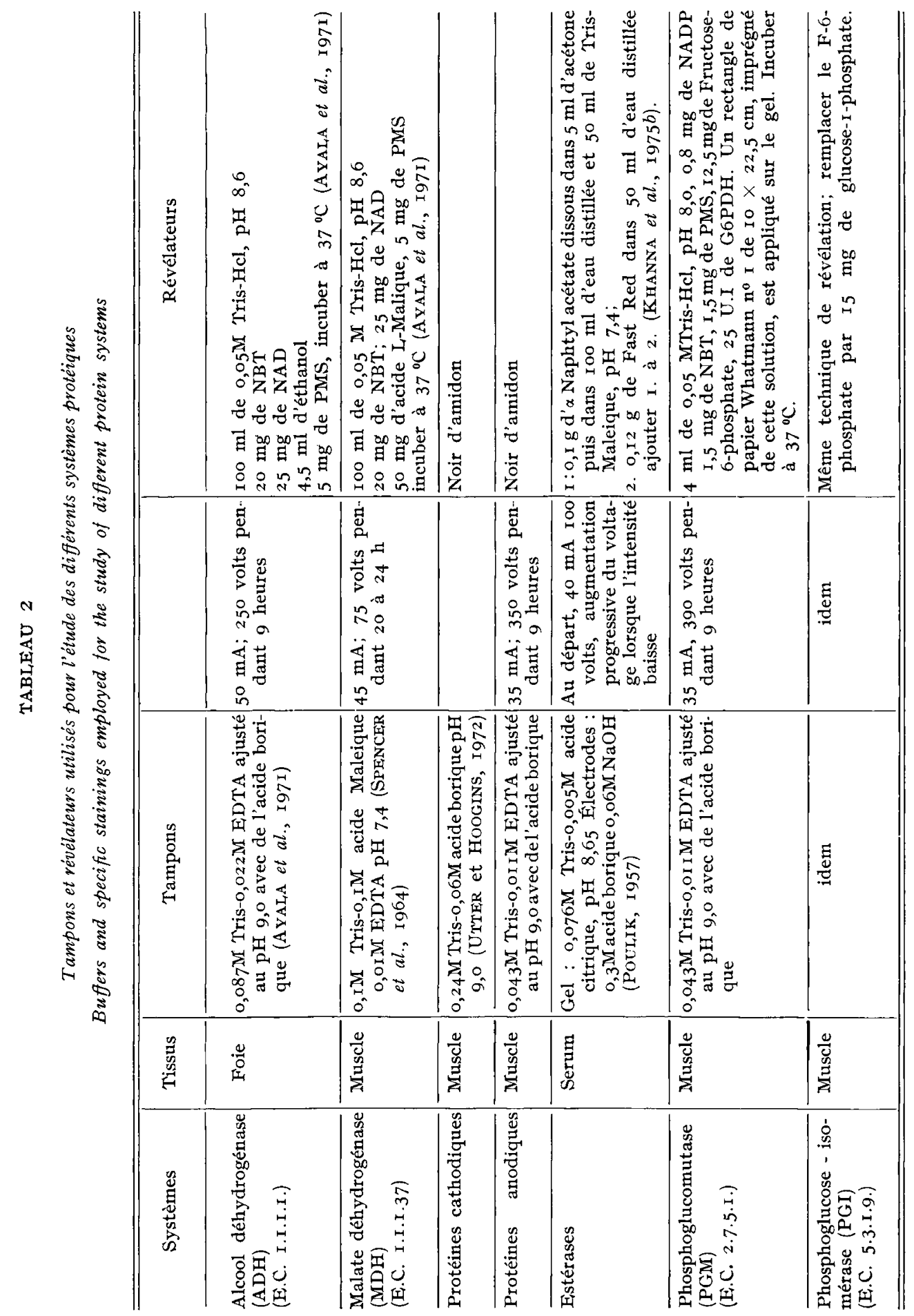


chaque croisement est indiqué dans le tableau $\mathrm{I}$. Chaque système a été étudié sur des alevins en cours de résorption de la vésicule vitelline et des adultes immatures de chaque espèce.

Les alevins hybrides sont issus des croisements suivants :

- Mâle Omble de fontaine (Salvelinus fontinalis Mitchell) $\times$ femelle Truite arc-en-ciel (Salmo gairdneri R.);

- Mâle Truite fario (Salmo trutta L.) $\times$ femelle Truite arc-en-ciel;

- Mâle Saumon Coho (Oncorhynchus kisutch Walbaum) $\times$ femelle Truite arcen-ciel;

- Mâle Omble de fontaine $\times$ femelle Truite fario;

- Mâle Truite fario $\times$ femelle Saumon atlantique (Salmo salar L.).

Ils ont été respectivement examinés 87,87, I27, I36 et I35 jours après 1a fécondation pour une température d'incubation de $10 \pm 2{ }^{\circ} \mathrm{C}$.

Les extraits tissulaires ont été préparés par broyage d'alevins entiers ou de tissus dans un tampon 0,05 M Tris-phosphate $-0,005 \mathrm{M} \mathrm{MgSO}_{4} \mathrm{pH} 7,4$ et centrifugation à $3000 \mathrm{~g}$ pendant $\mathrm{I}_{5}$ minutes.

Les gels à 12 p. Ioo d'amidon (Connaught) ont été coulés dans des moules de $6 \times 170 \times 225 \mathrm{~mm}$.

Les tampons, conditions d'électrophorèse et révélateurs particuliers à chaque système, sont indiqués dans le tableau 2 .

\section{Résultats et discussion}

\section{I. -- Étude des espèces parentales}

\section{I.I. - Systèmes ne présentant aucune variation intraspécifique.}

- Protéines anodiques du muscle; la figure I reproduit les électrophorégrammes obtenus.

- PGI : Chez les Truites fario et arc-en-ciel, l'Omble de fontaine et le Saumon coho, les électrophorégrammies à 6 bandes (fig. 2) peuvent être interprétés à partir des hypothèses d'Avise et KirTro (I973) selon lesquelles trois locus fixés synthétisent trois monomères différents, se combinant deux à deux pour former la molécule dimère de PGI.

Les Saumons atlantiques constituent une exception puisque trois bandes seulement apparaissent. Un modèle génétique basé sur deux locus fixés et codant deux sous-unités différentes qui s'associent deux à deux, permet d'expliquer les phénotypes observés.

Les résultats différents des nôtres ont parfois été rapportés. Les travaux de Khanna et al. (I975) sur le Saumon atlantique suggèrent l'existence de trois locus; ENGEL et al. (I977) ont obtenu chez la Truite fario des électrophorégrammes à trois bandes semblables à ceux des Saumons atlantiques que nous avons examinés.

Ces différences sont imputables aux techniques d'électrophorèse utilisées ou à d'éventuelles différences génétiques entre populations.

- Estérases : seules les estérases plasmatiques du Saumon atlantique et de la Truite fario ont été étudiées. Une seule bande d'activité estérasique apparaît 


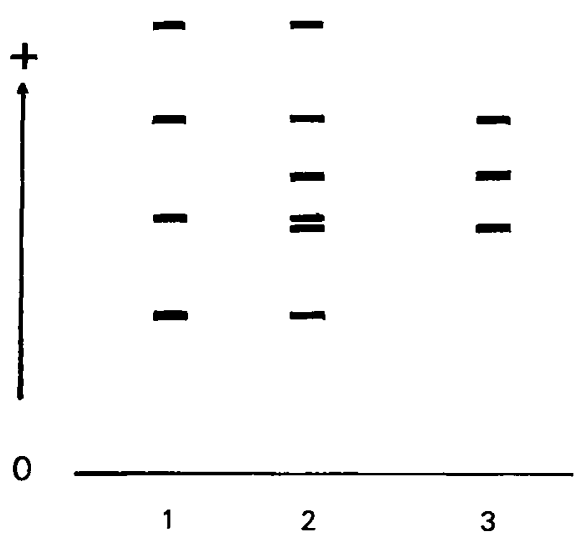

FIG. I. - Protéines anodiques du muscle: interprétation des électrophorégrammes

I : Saumon coho; 2 : Mâle Saumon Coho $x$ femelle Truite arc-en-ciel;

3 : Truite arc-en-ciel; Truite fario; Omble de fontaine.

Anodal muscle proteins : electrophoregram interpretation

I : Coho Salmon; 2 : Mâle coho Salmon $\times$ female rainbow Trout;

3 : rainbow Trout; brown Trout; brook Trout.

(fig. 3a). PAyne et al. (I972) ont utilisé l'estérase plasmatique pour identifier les hybrides naturels de ces deux espèces.

- ADH : L'ADH s'exprime dans le foie et migre vers la cathode. Elle est monomorphe chez les cinq espèces de salmonidés qui peuvent être réparties en deux groupes d'après la mobilité électrophorétique de cette enzyme (fig. $3 b$ ).

- Protéines cathodiques du muscle : la figure $3 c$ reproduit les électrophorégrammes obtenus, la truite fario différant des trois salmonidés d'origine américaine.

\section{I.2. - Systèmes présentant des variations alléliques.}

- MDH : Chez les Truites fario et arc-en-ciel, l'Omble de fontaine et le Saumon coho, nous avons constaté des variations intraspécifiques se traduisant pour chaque espèce, par trois types d'électrophorégrammes (cf. fig. 4a). BAILEY et al. (I970) ont démontré que deux locus $m d h$-BI et $m d h-\mathrm{B} 2$ codent la $\mathrm{MDH}$ du muscle de Truite arc-en-ciel et que des variations faisant intervenir deux allèles $a$ et $b$ se produisent à l'un des locus, $m d h-\mathrm{B} 2$. La forme fixée en $m d h$-BI et l'un des allèles de $m d h-\mathrm{B} 2$ synthétisant des sous-unités de même vitesse électrophorétique, sont considérés comme identiques. Les trois génotypes rencontrés peuvent donc s'écrire : $\frac{a a}{a a}$, $\frac{a a}{a b}$ et $\frac{a a}{b b}$; puisque $\mathrm{MDH}$ est une enzyme dimère, il en résulte les trois images électrophorétiques décrites, 1'une à bande unique, les deux autres constituées de trois bandes dont les intensités relatives sont dans le rapport 9-6-I chez les individus $\frac{a a}{a b}$ et I-2-I chez les homozygotes $\frac{a a}{a b}$. Ce modèle a été retrouvé chez le Saumon rose, Oncorhynchus gorbuscha (Aspinwal, I, I974; May et al., I975), 


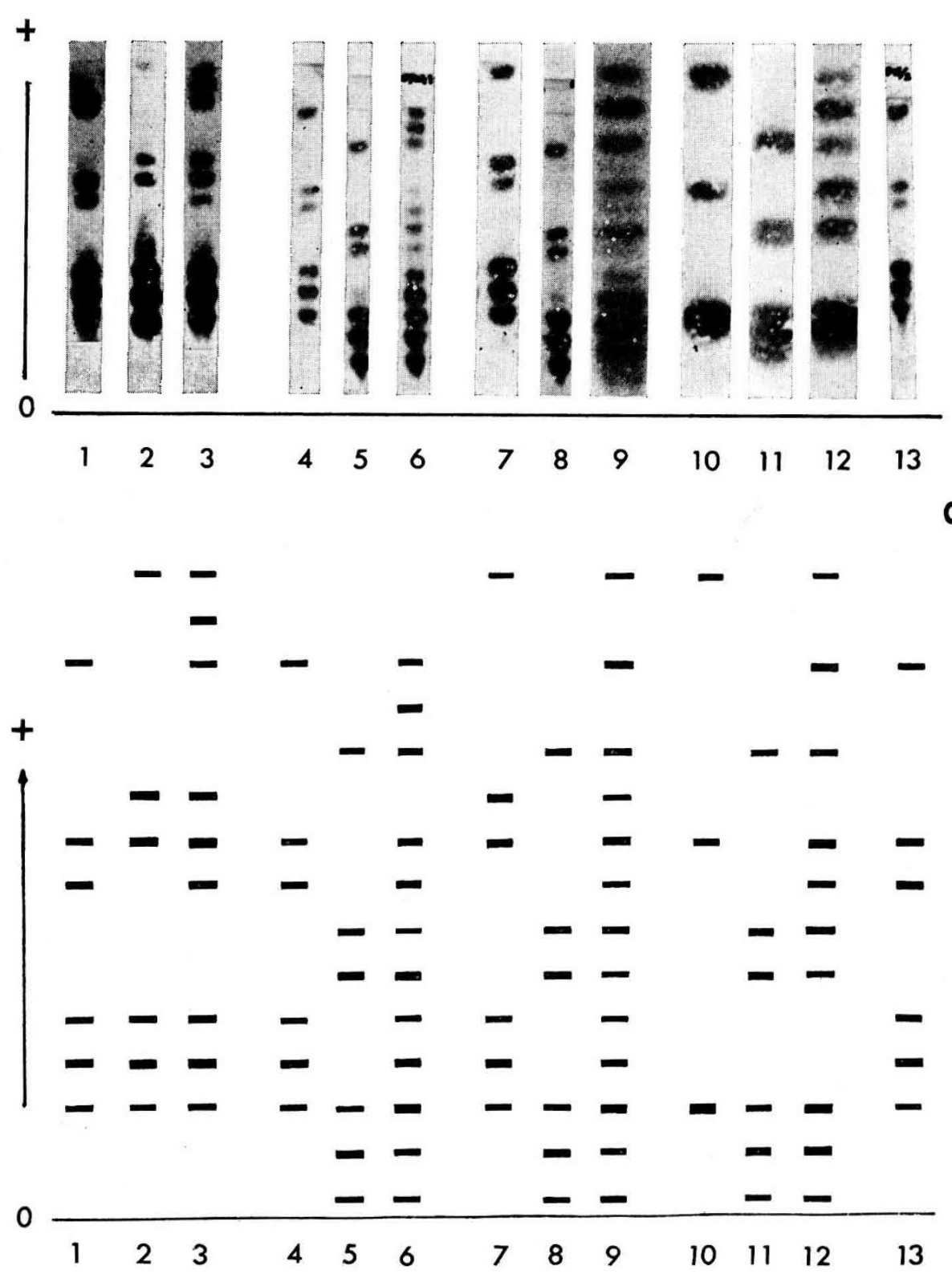

FIG. 2. - Phosphoglucose isomérase du muscle : electrophorégrammes et leurs interprétations.

a) Photographies des électrophorégrammes.

b) Interprétations.

I et 4 : Truite arc-en-ciel; 2 et $7:$ Omble de fontaine; $3:$ mâle Omble de fontaine $\times$ femelle Truite arc-en-ciel; 5,8 et I I : Truite fario; $6:$ mâle Truite fario $\times$ femelle Truite arc-en-ciel; 9 : mâle Omble de fontaine $\times$ femelle Truite fario; ro: saumon Atlantique; I 2 : mâle Truite fario $\times$ femelle Saumon atlantique; I3: Saumon Coho. 
le Saumon keta, Oncorhynchus keta (Numachi et al., I972; MAx et al., 1975), le Saumon coho, Oncorhynchus kisutch (UTTER et al., I973).

Les analogies que les électrophorégrammes de la Truite arc-en-ciel et du Saumon coho présentent respectivement avec ceux de la Truite fario et de l'Omble de fontaine nous ont conduit à supposer que les conclusions de BAILEY et al. sont également applicables à ces deux espèces. Les différences portent essentiellement sur l'allèle fixé en $m d h$-BI qui montre une mobilité égale à celle de 1'allèle rapide de $m d h-\mathrm{B} z$ chez les Truites fario et arc-en-ciel, et à celle de l'allèle lent chez le Saumon coho et 1'Omble de fontaine.

- PGM : Des variations allèliques affectent le locus PGM de la Truite arc-en-
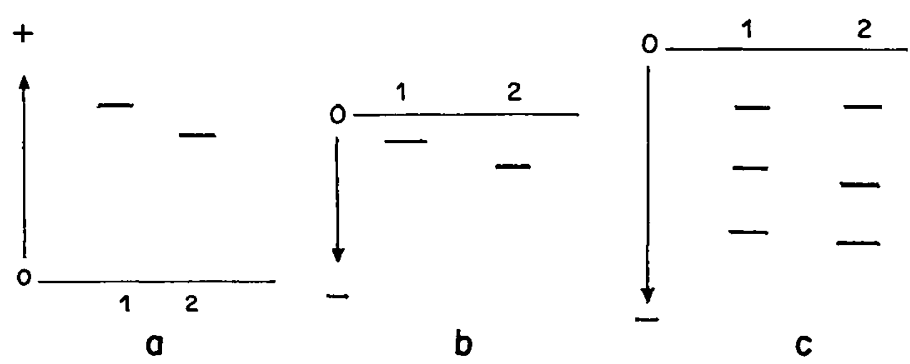

FIG. 3. - Interprétations des électrophorégrammes des estérases du sérum, de l'alcool déhydrogénase du foie et des protéines cathodiques du muscle.

$a$ : Estérases du sérum.

I : Saumon Atlantique; 2 : Truite fario.

$b$ : Alcooldehydrogenase du foie.

I : Truite arc-en-ciel, Saumon coho, Omble de fontaine; 2 : Truite fario, Saumon atlantique.

$c$ : Protéines cathodiques du muscle.

I : Truite arc-en-ciel; Omble de fontaine; Saumon coho; 2 : Truite fario.

Electrophoregrams interpretations of serum esterases, liver alcohol dehydrogenase and cathodal muscle proteins.

$a$ : Serum Esterases.

I : atlantic Salmon; 2 : brown Trout.

$b$ : Liver alcohol dehydrogenase.

I : Rainbow Trout, coho Salmon, brook Trout; 2 : brown Trout, atlantic Salmon.

$c$ : Cathodal muscle proteins.

I : Rainbow Trout, brook Trout, coho Salmon; 2 : brown Trout.

Suite de la page 22.

Muscle phosphoglucose isomerase: electrophoregrams and their interpretations.

a) Electrophoregrams pictures.

b) Interpretations.

I and 4 : rainbow Trout; 2 and $7:$ brook Trout; $3:$ mâle brook Trout $\times$ female rainbow Trout; 5, 8 and I I : brown Trout; 6 : mâle brown Trout $\times$ female rainbow Trout ;9: male brook $\times$ female brown Trout; Io : atlantic Salmon; i 2 : male brown Trout $\times$ female Atlantic Salmon; I3 : coho Salmon. 

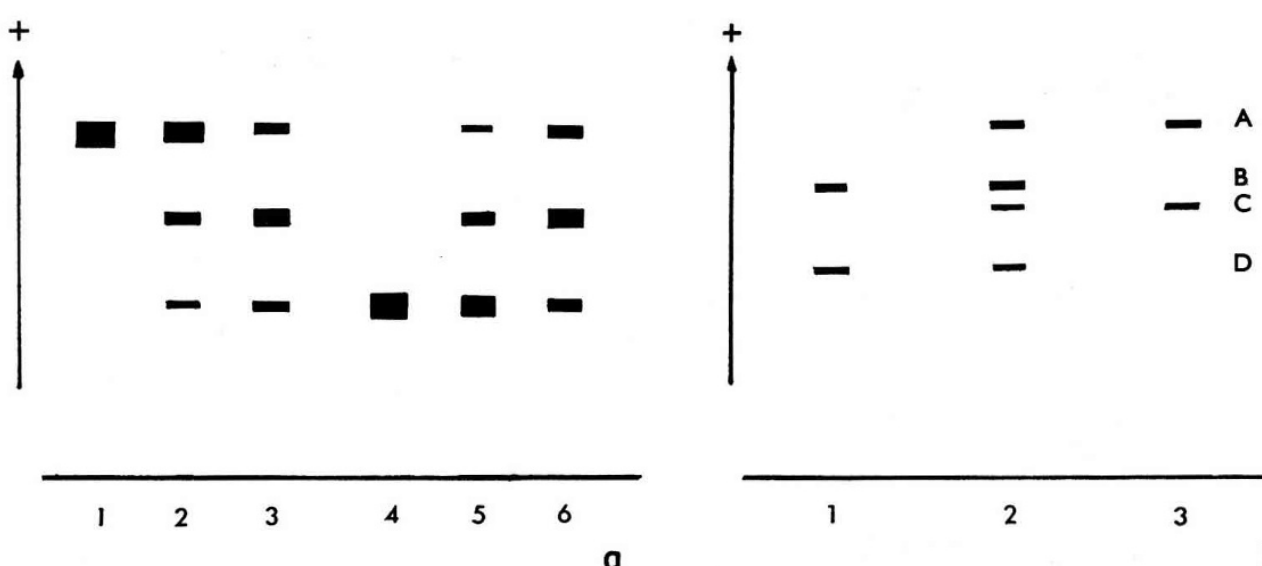

FIG. 4. - Interpretations des electrophorégrammes de deux enzymes du muscle : la malate dehydrogénase

a) Malate dehydrogénase. et la phosphoglucomutase.

I, 2 et 3 , Truite arc-en-ciel et Truite fario : I, $\frac{a a}{a a} ; 2, \frac{a a}{a b} ; 3, \frac{a a}{b b}$.

4,5 et 6 , Omble de fontaine et Saumon coho $: 4, \frac{b b}{b b} ; 5, \frac{b b}{a b} ; 6, \frac{b b}{a a}$.

b) Phosphoglucomutase.

I : Truite fario, Saumon coho et Truite arc-en-ciel (génotype $b b$ ); 2 : Truite arc-en-ciel (genotype $a b) ; 3$ : Truite arc-en-ciel (génotype $a a$ ), Omble de fontaine.

\section{Electrophoregrams Interpretations of two muscle enzyms: Malate dehydrogenase} and phosphoglucomutase.

a) Malate dehydrogenase.

I, 2 and 3, rainbow Trout and brown Trout : $1, \frac{a a}{a a} ; 2, \frac{a a}{a b} ; 3, \frac{a a}{b b}$.

4,5 and 6 , brook Trout and coho Salmon : $4, \frac{b b}{b b} ; 5, \frac{b b}{a b} ; 6, \frac{b b}{a a}$.

b) Phosphoglucomutase.

I : Brown Trout, coho Salmon and rainbow Trout (genotype $b b$ ); 2 :rainbow Trout (genotype $a b$ ); 3 : rainbow Trout (genotype $a a$ ), brook Trout.

ciel; l'un des phénotypes homozygotes est identique à celui de l'Omble de fontaine, l'autre à ceux de la Truite fario et du Saumon coho (fig. $4 b$ ). Les bandes C et D correspondraient à des formes déphosphorylées des bandes A et B (WIIKInson, I970).

\section{2. - Étude des hybrides.}

Compte tenu de la taille des alevins hybrides, seuls les systèmes s'exprimant dans le muscle ont été retenus. Pour chaque croisement, nous avons choisi un système différant d'une espèce à l'autre par la mobilité des bandes, mais ne présentant pas de variabilité in traspécifique, dans les populations dont nous disposions.

De ce fait, $\mathrm{MDH}$ et PGM ont dû être écartés puisque les variations intraspécifiques se traduisaient parfois chez deux espèces par des électrophorégrammes qualitativement semblables. Dans le cas de $\mathrm{MDH}$, les différences entre la Truite 
arc-en-ciel et le Saumon coho en $m d h$-BI sont masquées par les variations du locus $m d h-\mathrm{B} 2$.

La Truite arc-en-ciel et le Saumon coho ont été différenciés par les protéines anodiques du muscle, les Truites arc-en-ciel et fario, l'Omble de fontaine et le Saumon atlantique par la PGI. Ces deux systèmes sont présents et identiques chez 1'adulte et l'alevin, en fin de résorption, d'une même espèce.

Nous avons trois types de résultats :

- L'image électrophorétique de l'hybride représente la somme des électrophorégrammes parentaux; les électrophorégrammes des protéines anodiques des hybrides mâles Saumon coho $x$ femelle Truite arc-en-ciel entrent dans cette catégorie.

- Sur les électrophorégrammes des quatre autres croisements, entre les bandes parentales apparaissent des bandes supplémentaires dues à la combinaison de sous-unités produites par l'un et l'autre des parents.

- Trois individus, deux d'entre eux issus du croisement mâle Omble de fontaine $\times$ femelle Truite arc-en-ciel et le troisième d'un croisement mâle Saumon coho $\times$ femelle Truite arc-en-ciel, présentent des électrophorégrammes du type maternel. L'absence de l'une des bandes parentales a déjà été observée chez certains hybrides interspécifiques (OHNO et al. I968; PAYNE et al., 1972).

Les deux premières catégories correspondent à un mode d'expression codominant des gènes. Si 1'on exclut des erreurs de manipulation peu vraisemblables, deux interprétations peuvent être envisagées pour le troisième cas :

I. Les trois individus sont effectivement des hybrides, mais il n'est pas possible de les reconnaître pour l'une des raisons suivantes :

- Les locus permettant l'identification des hybrides sont polymorphes chez l'espèce paternelle. Bien que nous n'ayons décélé aucun allélisme aux locus PGI et protéines anodiques du muscle, cette hypothèse ne peut être rejetée puisque les parents n'ont pas été examinés.

- Les gènes paternels sont reprimés.

- D'autres phénomènes tels que des méioses défectueuses ou des mutations lors de la gamétogenèse peuvent être invoqués.

2. Les trois individus sont issus d'un développement gynogénétique. Ce mode de reproduction existe à l'état naturel chez les Poécilidés (UzELL, I970) et a été suggéré par UYENO (I972) pour certains croisements interspécifiques de Salmonidés. L'examen de plusieurs locus permettrait de choisir entre ces deux interprétations.

\section{Conclusion}

Nous avons donc pu vérifier le caractère hybride des individus issus des cinq croisements étudiés et nos résultats confirment l'intérêt des systèmes enzymatiques et protéiques pour caractériser les hybrides interspécifiques de première génération. L'identification des croisements $F_{2}$ ou des backcross semble plus délicate (NYMAN, I970), mais peut être réalisée en ayant recours à un plus grand nombre de gènes.

Une telle technique peut également être appliquée aux populations naturelles.

Les mécanismes d'isolement qui préservent dans certaines conditions de milieu, l'intégrité génétique des espèces, peuvent s'avérer inefficaces si l'environ- 
nement se dégrade ou lorsque sont introduites de nouvelles espèces (BEHN KE, I970). Il est donc important de disposer d'un outil qui permette de mettre en évidence les phénomènes d'introgression parfois bien avant l'apparition d'autres signes d'hybridation.

Reçu pour publication en avril 1978 .

\title{
Remerciements
}

Ce travail a été réalisé dans le cadre du contrat C.N.E.X.O. no $77 / 1619$ et de la convention I.N.R.A.-Ministère de la Qualité de la Vie ${ }^{\circ} 37 / 76$.

\section{Summary}

Electrophoretic identification of Salmonids hybrids.

\begin{abstract}
Five species of Salmonids, Brook Trout (Salvelinus fontinalis Mitchell), Rainbow Trout (Salmo gairdneri R.), Brown Trout (Salmo trutta L.), Coho Salmon (Oncorhynchus kisutch Walbaum) and Atlantic Salmon (Salmo salar L.) were electrophoretically studied for seven protein systems.

Fry from crosses Brook Trout male $\times$ Rainbow Trout female, Brown Trout male $\times$ Atlantic Salmon female, Brown Trout male $\times$ Rainbow Trout female, Brook Trout male $\times$ Brown Trout female were identified as hybrids by phosphoglucose isomerase (PGI); Anodal muscle proteins show that individuals from crosses coho Salmon male $\times$ Rainbow Trout female are hybrids.
\end{abstract}

\section{Références bibliographiques}

Aspinwal, N., I974. Genetic analysis of duplicate MDH loci in the pink Salmon Oncorhynchus gorbuscha. Genetics, 76, 65-72.

Avise J. C., KIT'To G. B., I972. Phosphoglucose isomerase gene duplication in the Bony fishes: An evolutionary history. Biochem. Genet., 8, I I3-132.

Ayala F., Powell J. R., Tracey M. L., Mourao C. A. et Perez-Salas S., i972. Enzyme variability in the Drosophila willistoni group. IV. - Genic variation in natural populations of Drosophila Willistoni. Genetics, 170, II3-139.

Bailey S. G., Wilson A. C., Halver J. E., Johnson C. L., I97o. Multiple forme of supernatant malate dehydrogenase in salmonid fishes. J. Biol. Chem., 245, 5927-5940.

BEHNKE R. J., I970. The application of cytogenetic and biochemical systematics to phylogenetic problem in family salmonidae. Trans. Am. Fish. Soc., 99, 237-248.

BILl,ARD R., PetiT J., JALABER'T B., SZÖLL̈̈SI D., I973. Artificial insemination in trout using a sperm diluant. Symposium on the early life history of fish. O.B.A.N., BLAXTER Ed., 7I 5-723.

Dangel J. R., Macy P. T., WiThler F. C., I973. Annotated bibliography of interspecific hybridization of fishes of the subfamily salmonidae. NOAA Technical Memorandum NMFS. NWFC I.

ENGEL W., KUhL, P., SchmidTKE J., I977. Expression of the paternally derived phosphoglucose isomerase genes during hybrid trout development. Comp. Biochem. Physiol., 56B, I03-I08.

Khanna N. D., Kumar Juneja R., LARsson B., Gahne B., 1975a. Electrophoretic studies on proteins and enzymes in the atlantic Salmon, Salmo salar L. Swedish. J. Agric. Res., 5, I85I92.

Khanna N. D., Kumar Juneja R., Larsson R., 1975b. Electrophoretic studies on esterases in the atlantic Salmon, Salmo salar L. Swedish J. Agric. Res., 5, 193-197.

MAY B., UTTER F. M., AlLENDORF F. W., 1975. Biochemical genetic variation in pink and chum Salmon. J. Heredity, 66 227-232. 
Numachi K., Matsumiya Y., SATE R., 1972. Duplicate genetic loci and variant forms of malate dehydrogenase in chum Salmon and rainbow Trout. Bull. Jap. Soc. Sci. Fish., 38, 699-7o6.

Nyman L., 1970. Electrophoretic analysis of hybrids between Salmon (Salmo salar L.) and Trout (Salmo trutta L.). Trans. Am. Rish. Doc., 99, 229-236.

Ohno S., CASTro SIERra E., I968. Allele inhibition at the autosomally inherited gene locus for liver alcohol dehydrogenase in Chicken-quail hybrids. Biochemical Genetics, 1, 323-335.

PAyne R. H., Child A. R., Forrest A., I972. The existence of natural hybrids between the European trout and the atlantic Salmon. J. Fish. Biol., 4, 233-236.

SchwarTz F. J., r972. World literature to fish hybrids with an analysis by family, species and hybrids. Publication of the Gulf Coast. Research laboratory, $\mathrm{n}^{0} 3$.

Spencer N., Hopkinson D. A., Harris H., 1964. Phosphoglucomutase polymorphism in man. Nature (Lond.), 204, 742.

Suzuki R., Fukuda Y., r973. Bull Freswater. Fish. Res. Lab., 23, nº $\mathbf{1}$, 5-32.

UTTER F. M., AlLENDORF F. W., Hodgins H. O., I974. Biochemical genetic studies of fishes: Potentialities and limitations in biochemical and biophysical perspectives. In: MarineBiology, Vol. I, p. 213-238; Eds by D.C. MaLins and J. R. SARgent. Academy Press.

UTTER F. M., AlLendorf F. W., Hodgins H. O., I973. Genetic variability and relationships in pacific Salmon and related Trout based on protein variations. Syst. Zool., 22, 257-270.

UYENO T., I972. Chromosomes of offspring resulting from crossing coho Salmon and brook Trout. Japan. J. Ichthyol., 9, I66-I 7I.

UZELI T., I970. Meiotic mechanisms of naturally occuring unisexual vertebrates. Nature (Lond.), 104, 433-445.

Wilkinson J. H., I970. Isoenzymes, $2^{\mathrm{e}}$ édition, Chapman and Hall Ltd, Londres (Ioo). 